Quality improvement by introduction of 72-hour admission pathway in Forest House Adolescent Unit (FHAU) for young people in crisis

Sadaf Mufti* and Linda Zirinsky

HPFT

${ }^{*}$ Corresponding author.

doi: 10.1192/bjo.2021.566

Aims. Short admissions for crisis management among young people suffering with Emotionally Unstable Personality Disorder (EUPD) as recommended in National Institute for Health and Care Excellence (NICE) 2009 guidelines are not routinely offered in the United Kingdom (UK). Our aim was to introduce crisis admissions lasting for 72 hours. During this brief admission the families of young people presenting with suicidal behaviour are offered an assessment and diagnosis of young person's difficulties, psychoeducation, and safety plan for future risky behaviour, in addition to respite.

Background. Three-day Crisis admission was set up with the aim of reducing inappropriate long admissions in people who may have more negative effects from admission than positive ones. A need was felt for a brief admission pathway in order to be able to provide treatment for patients suffering from EUPD traits in keeping with NICE guidelines. NICE guidelines suggest that people with borderline personality disorder should be considered for acute psychiatric inpatient admission only for the management of crises involving significant risk to self or others that cannot be managed by other services. The guidelines also recommend ensuring that the decision is based on an explicit and joint understanding of the potential benefits and likely harm that may result from admission and agreeing to the length and purpose of the admission in advance.

Method. A retrospective study comparing length of hospital stay in the 2018 (when this model was introduced) with previous years, the number of serious incidents was carried out to assess the impact of this new admission model. The rate of readmissions in the same year was also assessed. For qualitative feedback regarding the effectiveness of the crisis admission as an intervention, a survey was carried out to assess parent satisfaction and the nursing staff was asked for their views.

Result. There was a marked reduction in the number of serious incidents linked to suicide and length of hospital stay was reduced to half in the year when the crisis admissions were introduced as compared to the previous year. Only about $10-15 \%$ of patients required re-admission in the same year. About $90 \%$ of parents gave a positive feedback confirming the effectiveness of this intervention.

Conclusion. 72-hour crisis admissions for adolescents are effective, appropriate, clinically indicated alternative to routine admissions with a high parent satisfaction.

\section{Bridging the gap: improving liaison psychiatry documentation quality to meet the National Confidential Enquiry into Patient Outcome and Death (NCEPOD) treat as one recommendations at newcastle hospitals}

David $\mathrm{Ou}^{1 *}$, Sara Ibrahem ${ }^{1}$, Sahar Basirat ${ }^{2}$ and Sarah Brown ${ }^{2}$

${ }^{1}$ The Newcastle upon Tyne Hospitals NHS Foundation Trust and ${ }^{2}$ Cumbria, Northumberland, Tyne And Wear NHS Foundation Trust ${ }^{\star}$ Corresponding author.

doi: 10.1192/bjo.2021.567

Aims. This project aimed to assess and improve the quality and frequency of documentation from Psychiatric Liaison Team
(PLT) to ward-based medical colleagues against the Treat as One recommendations. From experience, we hypothesised that written documentation of information crucial to patient care is not consistently meeting standards. This communication breakdown directly affects patient safety, potentially introducing additional risks to our already vulnerable patient group.

Effective communication between PLT and our medical colleagues bridges the gap in providing continuity of care and ensures patients' mental and physical health needs are met in acute trusts. The NCEPOD found that there remains many barriers to high quality mental healthcare provided to patients in general hospitals and recommended 7 elements that PLT documentations should encompass.

Method. We audited initial PLT assessments and the resulting documentation to determine if these met the 7 standards set by NCEPOD. Baseline audit undertaken from 21-27/09/2020 encompassing 130 patient referrals to PLT.

A period of time was allotted to implement robust changes to improve the service. This included a streamlined e-template that automatically populates in the acute hospital eRecord system which prompts clinicians to document according to the NCEPOD standards, structured clinician training and education, and the nomination of "Treat as One Guardians" in the team to ensure that acute trust documentations are present during daily multidisciplinary meetings.

The cycle was then completed on 22-28/02/2021 with a re-audit capturing 55 referrals.

Result. Implementation of our recommended changes saw an increase from $58 \%$ of documentations with $\geq 50 \%$ NCEPOD elements to $98 \%$ in the re-audit.

We also saw an increase in number of the NCEPOD 7 elements included following intervention: formulation (0\% to $8 \%)$, legal status and capacity ( $47 \%$ to $79 \%)$, risk assessment ( $2 \%$ to $28 \%$ ), risk management ( $18 \%$ to $53 \%$ ), and discharge plan ( $2 \%$ to $29 \%)$.

Completion rate of acute trust documentation increased from $74 \%$ to $96 \%$.

Our interventions also led to more contemporaneous communication, significantly reducing mean time from assessment to documentation in both acute trust and mental health records from 6.02 to 3.53 hours, $(\mathrm{p}=0.04)$ and 6.12 to 3.50 hours, $(\mathrm{p}=$ $0.05)$ respectively.

Conclusion. Following our interventions, the results showed improving trends in the frequency and quality of our documentation with secondary outcomes showing increased documenting efficiency. Our current practice is not yet optimal and retains potential to adversely affect our patients. We propose further investigating barriers to change using the quality improvement PDSA (Plan, Do, Study, Act) methodology to continue innovating.

\section{Remote psychotropic medication advice for general practitioners: a quality improvement project}

Emily Rackley* and Rosemary King

Gloucestershire Health and Care NHS Foundation Trust ${ }^{\star}$ Corresponding author.

doi: 10.1192/bjo.2021.568

Aims. Our first aim was to first find out how confident general practitioners were about referring in to the Gloucester Recovery Team and managing psychotropic medications. Our second aim was to then improve general practitioner's self-rated scores of confidence in managing psychotropic medication 